\title{
On the Minimum Modulus of Analytic Functions of Moderate Growth in the Unit Disc
}

\author{
I. Chyzhykov ${ }^{1,2} \cdot$ M. Kravets ${ }^{1}$
}

Received: 25 July 2014 / Revised: 19 September 2014 / Accepted: 10 March 2015 /

Published online: 15 May 2015

(C) The Author(s) 2015. This article is published with open access at Springerlink.com

\begin{abstract}
We study the behavior of the minimum modulus of analytic functions in the unit disc in terms of $\rho_{\infty}$-order, which is the limit of the orders of $L_{p}$-norms of $\log \left|f\left(r e^{i \theta}\right)\right|$ over the circle as $p \rightarrow \infty$. This concept coincides with the usual order of the maximum modulus function if the order is greater than one. New results are obtained for analytic functions of order smaller than 1 .
\end{abstract}

Keywords Analytic function - Minimum modulus - Order of growth · Factorization · Zero distribution $\cdot$ Canonical product $\cdot$ Harmonic function

Mathematics Subject Classification $\quad 30 \mathrm{~J} 99 \cdot 30 \mathrm{H} 05 \cdot 30 \mathrm{H} 15 \cdot 30 \mathrm{~J} 10$

\section{Introduction and Main Results}

Let $\mathbb{D}_{R}=\{z \in \mathbb{C}:|z|<R\}, 0<R \leq \infty$, and $\mathbb{D}=\mathbb{D}_{1}$. For an analytic function $f$ on $\mathbb{D}_{R}$, we define the minimum modulus

$$
\mu(r, f)=\min \{|f(z)|:|z|=r\}, \quad 0<r<R,
$$

Communicated by James K. Langley.

$\bowtie \quad$ I. Chyzhykov

chyzhykov@yahoo.com

M. Kravets

marjana_s@ukr.net

1 Faculty of Mechanics and Mathematics, Ivan Franko National University of Lviv, Universytets'ka 1, Lviv 79000, Ukraine

2 Present Address: Faculty of Mathematics and Natural Studies, Cardinal Stefan Wyszyński University in Warsaw, Wóycickiego 1/3, 01-938 Warszawa, Poland 
and the maximum modulus

$$
M(r, f)=\max \{|f(z)|:|z|=r\}, \quad 0<r<R .
$$

Interplay between $\mu(r, f)$ and $M(r, f)$ has been studied in a large number of papers. In the case of entire functions, i.e., $R=\infty$, a survey of results up to 1989 can be found in Hayman's book ([16, Chap. 6]).

The orders of the growth of an analytic function $f$ in $\mathbb{D}_{\infty}$, and in $\mathbb{D}$, respectively, are defined as

$$
\rho[f]=\limsup _{r \nearrow \infty} \frac{\log ^{+} \log ^{+} M(r, f)}{\log r}, \quad \rho_{M}[f]=\limsup _{r \nearrow 1} \frac{\log ^{+} \log ^{+} M(r, f)}{-\log (1-r)} .
$$

For entire functions of order $\rho[f] \leq 1$, there are a lot of sharp results on the behavior such as $\cos \pi \rho$-theorem $([1,16])$.

Theorem ([1]) Suppose that $0 \leq \rho<\alpha<1$. If $f$ is an entire function of order $\rho$ and $f(z) \not \equiv$ const then

$$
\log \mu(r, f) \geq \cos \pi \alpha \log M(r, f), r \in E
$$

where

$$
\lim _{r \rightarrow \infty} \frac{\int_{E \cap[1, r)} \frac{d t}{t}}{\log r} \geq 1-\frac{\rho}{\alpha}
$$

One of the most interesting open problems for entire functions of order greater than 1 is to find the asymptotic behavior of the minimum modulus with respect to the maximum modulus, especially for values of $\rho[f]$ close to $1([14,15])$. The most precise results concerning the minimum modulus of entire and subharmonic functions of order zero can be found in [2-4,11-13].

For analytic functions in the unit disc $\mathbb{D}$ the situation, in a certain sense, is the opposite. Known results are much weaker in accuracy than the statements of the $\cos \pi \rho$-theorem type. Moreover, these results mainly concern analytic functions with $\rho_{M}[f] \geq 1$.

We start with an old result of M. Heins.

Theorem A [18] If $f(z)$ is analytic in $\mathbb{D}, f(z) \not \equiv$ const, $f(z)$ is bounded in $\mathbb{D}$, then there exist a constant $K>0$ and a sequence $\left(r_{n}\right), r_{n} \nearrow 1$ such that

$$
\log \mu\left(r_{n}, f\right) \geq-\frac{K}{1-r_{n}}, n \rightarrow+\infty
$$

For the function $f(z)=\exp \left(\frac{1}{z-1}\right)$, we have

$$
\log M(r, f)=O(1), \log \mu(r, f)=-\frac{1}{1-r}, \quad r \nearrow 1
$$


Thus, inequality (1) is sharp in the class of bounded analytic functions in the unit disc. A description of exceptional sets for the relation $(1-|z|) \log |B(z)| \rightarrow 0,|z| \nearrow 1$, where $B$ is a Blaschke product, has been very recently obtained in [17].

In the general case, we have the following theorem of C.N. Linden.

Theorem $\mathbf{B}[20]$ Let $f(z)$ be an analytic function, $f(z) \not \equiv$ const in $\mathbb{D}, \rho=\rho_{M}[f]>$ 1 , then there is a constant $K(\rho)$ such that

$$
\log \mu\left(r_{n}, f\right)>-K(\rho) \log M\left(r_{n}, f\right) \log \log M\left(r_{n}, f\right)
$$

for some sequence of number $\left(r_{n}\right), r_{n} \nearrow 1$.

The following theorem plays a key role for the estimates of minimum modulus.

Theorem $\mathbf{C}$ [20] Let $f$ be analytic in $\mathbb{D}$, and suppose that $\frac{1}{2} \leq \alpha<1$. Then, there exists $R_{0}=R_{0}(\alpha) \in(0,1)$ such that for arbitrary $R \in\left[R_{0}, 1\right)$, there is a set $E_{R} \subset\left[R^{2}, R\left(R+\frac{1}{16}(1-R)\right)\right]$ of measure at least $\frac{1}{32} R(1-R)$ such that

$$
\begin{aligned}
\log \mu(r, f) \geq & -\frac{C}{(1-R)^{\frac{1}{\alpha}}} \log \frac{1}{1-R} \\
& \times\left(\int_{0}^{R} \log ^{+} M(t, f)(R-t)^{\frac{1}{\alpha}-1} d t+\log ^{+} M\left(R_{0}, f\right)\right),
\end{aligned}
$$

$r \in E_{R}, C=C\left(\alpha, R_{0}\right)>0$.

Such an approach for analytic functions $f$ of order $\rho_{M}[f]<1$ allows us to get the following results.

Theorem $\mathbf{D}$ [22] Let $0 \leq \rho_{M}[f]<1, f(z)$ be an analytic in $\mathbb{D}, f(0)=1$ and

$$
\log M(r, f)<A(1-r)^{-\rho_{M}[f]}, 0 \leq r<1 .
$$

Then, there exist $R_{1} \in(0,1)$ depending on $\rho_{M}$ and $K=K\left(A, R_{1}\right)$ such that, if $R \in\left(R_{1}, 1\right)$, then the interval $\left(R, \frac{1}{2}(1+R)\right)$ contains a set of values $r$ of measure at least $\frac{1}{4}(1-R)$ such that

$$
\log \mu(r, f)>-\frac{K}{1-r} \log \frac{1}{1-r} .
$$

Let $\left(a_{n}\right)$ be a sequence of zeros of analytic function $f$ in $\mathbb{D}$. For this sequence, we define

$$
n_{z}(t)=\sum_{\left|a_{n}-z\right| \leq t} 1
$$

Theorem $\mathbf{E}$ [22] Let $f(z)$ be analytic in $\mathbb{D}, f(z) \not \equiv$ const, $\rho_{M}[f]<1$. If there are $r_{0} \in(0,1)$ and a constant $B$ such that 


$$
n_{r e^{i \theta}}\left(\frac{1-r}{2}\right)<\frac{B}{(1-r) \log \left(\frac{1}{1-r}\right)}, 0 \leq \theta<2 \pi, r_{0} \leq r<1 \text {, }
$$

then there exist $K>0, L \geq \frac{1}{4}, \rho_{0} \in(0,1)$ such that, if $R \in\left(\rho_{0}, 1\right)$, then the interval $\left(R, \frac{1}{2}(1+R)\right)$ contains a set $r$ of measure at least $L(1-R)$ such that

$$
\log \mu(r, f)>-\frac{K}{1-r}
$$

A characteristic feature of Theorems $\mathrm{D}$ and $\mathrm{E}$ is that their conclusions do not depend on the corresponding value of order $\rho_{M}[f] \in[0,1]$. It appears that, in this case, the value $\rho_{M}[f]$ does not allow the behavior of the minimum modulus in terms of conditions on zeros of $f$ to be described more precisely. The aim of this paper is to correct this defect. Note that, some classes of bounded analytic functions satisfying the inequality

$$
\log \mu(r, f) \geq-\frac{K}{(1-r)^{\alpha}}, \quad 0<\alpha<1,
$$

were found in [5].

For an analytic function $f(z), z \in \mathbb{D}, f \not \equiv 0$ and $p \geq 1$, we define

$$
m_{p}(r, f)=\left(\left.\frac{1}{2 \pi} \int_{0}^{2 \pi}|\log | f\left(r e^{i \theta}\right)\right|^{p} d \theta\right)^{\frac{1}{p}}, \quad 0<r<1
$$

We write

$$
\rho_{p}[f]=\lim \sup _{r \nearrow 1} \frac{\log m_{p}(r, f)}{-\log (1-r)} .
$$

We define the order $\rho_{\infty}[f]$ of the function $f$ as

$$
\rho_{\infty}[f]=\lim _{p \rightarrow+\infty} \rho_{p}[f] .
$$

The limit exists since $\rho_{p}$ is a non-decreasing function in $p$ ([24]). This quantity appeared for the first time in a work of Linden [23], who proved that $\rho_{M}[f]=\rho_{\infty}[f]$ provided that $\rho_{M}[f]>1$, and $\rho_{M}[f] \leq \rho_{\infty}[f]$, but he did not study the classes of functions defined by the order $\rho_{\infty}[f]$ when $\rho_{\infty}[f]<1$. Applications of this concept to factorization of analytic functions in $\mathbb{D}$, and logarithmic derivative estimates can be found in $[6,9]$.

Let a sequence $\left(a_{n}\right)$ in $\mathbb{D}$ satisfy the condition

$$
\sum_{n}\left(1-\left|a_{n}\right|\right)^{s+1}<+\infty, \quad s \in \mathbb{Z}_{+}
$$


Consider the canonical product, $s \in \mathbb{N}$,

$$
P(z)=\prod_{n=1}^{\infty} E\left(A_{n}(z), s\right)
$$

where $E(w, 0)=1-w$,

$$
E(w, s)=(1-w) \exp \left\{w+w^{2} / 2+\ldots+w^{s} / s\right\}, \quad s \in \mathbb{N},
$$

is the Weierstrass primary factor, and $A_{n}(z)=\frac{1-\left|a_{n}\right|^{2}}{1-\bar{a}_{n} z}$. The function $P(z)$ is analytic in the unit disc with the zero sequence $\left(a_{n}\right)$ provided that $(2)$ holds. We note that if $s=0$, we have $P_{0}(z)=C B(z)$, where $C=\prod_{n}\left|a_{n}\right|$,

$$
B(z)=\prod_{n} \frac{\bar{a}_{n}\left(a_{n}-z\right)}{\left|a_{n}\right|\left(1-\bar{a}_{n} z\right)}
$$

is the Blaschke product corresponding to the sequence $\left(a_{n}\right)$ provided that $\sum_{n}(1-$ $\left.\left|a_{n}\right|\right)<\infty$. We define

$$
N_{z}(h)=\sum_{\left|a_{n}-z\right| \leq h} \log \frac{h}{\left|z-a_{n}\right|}=\int_{0}^{h} \frac{n_{z}(s)}{s} d s, \quad 0<h<1-|z| .
$$

Let $E \subset[0,1)$ be a measurable set. The upper density of $E$ is defined by

$$
D_{1}(E)=\limsup _{r \nearrow 1} \frac{\lambda_{1}(E \cap[r, 1))}{1-r}
$$

where $\lambda_{1}(E \cap[r, 1))$ denotes the Lebesgue measure of $E \cap[r, 1)$. Theorem 1 describes the minimum modulus of canonical products of genus $s \in \mathbb{N}$.

Theorem 1 Given a sequence $\left(a_{n}\right)$ in $\mathbb{D}$, suppose that $n_{z}\left(\frac{1-|z|}{2}\right) \leq\left(\frac{1}{1-|z|}\right)^{\beta}$, for some $\beta>0$, and all $z \in \mathbb{D} \backslash \mathbb{D}_{r_{0}}, 0 \leq r_{0}<1$, and let $P(z)$ be the canonical product of genus $s \geq[\beta]+1$ with zeros $\left(a_{n}\right)$. Then, for arbitrary $K_{1}, K_{2}>1$, there exist a constant $C \in\left(0, \frac{2}{3}\right]$ and a set $F \subset[0,1)$ such that

$$
N_{z}\left(\frac{1-|z|}{4}\right) \leq K_{1}\left(\frac{1}{1-|z|}\right)^{\beta} \log \frac{1}{1-|z|}
$$

and

$$
\log \mu(r, P) \geq-K_{2} \frac{1}{(1-r)^{\beta}} \log \frac{1}{1-r}, \quad r \in[0 ; 1) \backslash F,
$$

where $D_{1}(F) \leq C$. 
Remark 1 An example from [20, Theorem 6] shows that for all $\beta \geq 1$ there exists an analytic function in $\mathbb{D}$ satisfying the conditions of Theorem 1 , and of order $\rho_{M}[f]=\beta$ such that

$$
\log \mu(r, f) \leq-K_{3} \frac{1}{(1-r)^{\beta}} \log \frac{1}{1-r},
$$

holds for all $r \in[0,1)$ and some constant $K_{3}(\beta)>0$.

In the general case, we need the following factorization theorem.

Theorem $\mathbf{F}$ [6] Let $f$ be an analytic function in $\mathbb{D}$, and of finite order $\rho_{\infty}[f]$. Then,

$$
f(z)=z^{p} P(z) g(z)
$$

where $P(z)$ is a canonical product of form (3) displaying the zeros of $f, p$ is a nonnegative integer, $g$ is non-zero and both $P$ and $g$ are analytic, and $\rho_{\infty}[P] \leq \rho_{\infty}[f]$, $\rho_{\infty}[g] \leq \rho_{\infty}[f]$.

Let $u(z)$ be a harmonic function in $\mathbb{D}$. We then define

$$
m_{p}(r, u)=\left(\frac{1}{2 \pi} \int_{0}^{2 \pi}\left|u\left(r e^{i \theta}\right)\right|^{p} d \theta\right)^{\frac{1}{p}}
$$

Denote $M_{\infty}(r, u)=\max \{|u(z)|:|z|=r\}$. The following statement is of some independent interest. It gives another way to compute $\rho_{\infty}$-order of an analytic function without zeros.

Proposition 1 Let $u(z)$ be a harmonic function in $\mathbb{D}$. Then, we have

$$
\rho_{\infty}[u]=\limsup _{r \nearrow 1} \frac{\log M_{\infty}(r, u)}{-\log (1-r)} .
$$

The main result of this paper is the following.

Theorem 2 Let $f$ be analytic in $\mathbb{D}, \rho_{\infty}[f]=\rho, \rho<+\infty$. Then, for arbitrary $\varepsilon>0$, there exists $C \in(0,1)$, and a set $F \subset[0,1)$, such that

$$
\log \mu(r, f) \geq-\frac{1}{(1-r)^{\rho+\varepsilon}}
$$

for $r \in[0,1) \backslash F, D_{1}(F) \leq C$.

Remark 2 Theorem 2 gives us substantially new information when $\rho_{\infty}[f]<1$.

Remark 3 The function $g(z)=\exp \left\{-\frac{1}{(1-z)^{\alpha}} \log \frac{1}{1-z}\right\}, g(0)=1$, shows that $\varepsilon$ in the inequality (5) cannot be omitted.

Some generalizations of Theorems 1 and 2 are considered in Sect. 3. 


\section{Proof of the Main Results}

The following lemma is important in our investigation.

Lemma 1 [21] For a given value $\theta$ let $S_{m, k}$ denote the region

$$
1-2^{-k} \leq|z|<1-2^{-k-1}, \quad 2 \pi m 2^{-k}<\theta-\arg z \leq 2 \pi(m+1) 2^{-k},
$$

where $k$ and $m$ are integers such that $k>0$ and $-2^{k-1} \leq m \leq 2^{k-1}-1$. Let $k_{0}$ be a positive integer and $\beta>0$. Suppose that there are a finite number of points $a_{n}$ in $\left\{|z|<1-2^{-k_{0}}\right\}$ and that for some value $\theta$ such that $0 \leq \theta<2 \pi$ there are at most $C 2^{k \beta}$ points $a_{n}$ in each region (6) for $k \geq k_{0}$. Then, if $s$ is an integer greater than $\beta$ the function $P$ defined by (3) is analytic in $\mathbb{D}$ and

$$
\log |P(z)| \leq 2^{s+2} \sum_{n=1}^{\infty}\left|\frac{1-\left|a_{n}\right|^{2}}{1-z \bar{a}_{n}}\right|^{s+1}<K(1-|z|)^{-\beta}
$$

where $K$ depends on $s, \beta, C$.

Proof of Theorem 1 Without loss of generality, we may assume that $r_{0}=0$. Otherwise,

$$
n_{z}\left(\frac{1-|z|}{2}\right) \leq \frac{C_{r_{0}}}{(1-|z|)^{\beta}}
$$

where $C_{r_{0}}=\max \left\{1, n_{0}\left(\frac{1+r_{0}}{2}\right)\right\}$, because $n_{z}\left(\frac{1-|z|}{2}\right) \leq n_{0}\left(\frac{1+r_{0}}{2}\right)$ if $|z| \leq r_{0}$. Since each $S_{m, k}$ can be covered by a uniformly bounded number of discs of the form $\left\{\zeta:|\zeta-z|<\frac{1-|z|}{2}\right\}$ with the centers in $S_{m, k}$, the assumptions of Lemma 1 are satisfied. Hence, the inequality $n_{z}\left(\frac{1-|z|}{2}\right) \leq\left(\frac{1}{1-|z|}\right)^{\beta}$ holds in $\mathbb{D}$ and we have from Lemma 1

$$
\sum_{k=1}^{\infty}\left|A_{k}(z)\right|^{s+1} \leq K\left(\frac{1}{1-r}\right)^{\beta}
$$

We denote $r_{N}=1-\left(\frac{3}{4}\right)^{N}$, and use the following lemma.

Lemma 2 [15] Suppose that $r>0, h>0$ and that for $|z|=r$ we have $n_{z}(h) \leq n_{0}$. Then there exist a set $\mathscr{E} \subset\left[r, r+\frac{h}{2}\right]$ having measure at least $\frac{1}{4} h$ such that, for $R$ in $\mathscr{E}$ and $|z|=R$,

$$
N_{z}\left(\frac{h}{2}\right) \leq n_{0} \log \frac{A(r+h)}{h}
$$

where A is an absolute constant. 
We apply this lemma with $r=r_{N}, h=\frac{1}{2}\left(1-r_{N}\right)=\frac{1}{2}\left(\frac{3}{4}\right)^{N}$, and $n_{0}=\left(\frac{4}{3}\right)^{N \beta}$. Since $r_{N}+\frac{h}{2}=r_{N+1}$, there exists a set $\mathscr{E}_{N} \subset\left\{R: 1-r_{N} \leq R \leq 1-r_{N+1}\right\}$ such that $\lambda_{1}\left(\mathscr{E}_{N}\right) \geq \frac{1}{8}\left(\frac{3}{4}\right)^{N}$, and for $R \in \mathscr{E}_{N}$ and $|z|=R$ we obtain

$N_{z}\left(\frac{1}{2} h\right) \leq\left(\frac{4}{3}\right)^{\beta N} \log \frac{A\left(r_{N}+h\right)}{h} \leq\left(\frac{1}{1-r_{N}}\right)^{\beta} \log \frac{2 A}{1-r_{N}} \leq\left(\frac{1}{1-R}\right)^{\beta} \log \frac{2 A}{1-R}$

We define $F=\bigcup_{N=1}^{\infty} F_{N}$ where $F_{N}=\left[1-r_{N} ; 1-r_{N+1}\right] \backslash \mathscr{E}_{N}$. Let us prove that

$$
\lambda_{1}([r, 1) \cap F) \leq C(1-r)
$$

holds, where $0<C \leq \frac{2}{3}$. Note that,

$$
\lambda_{1}\left(F_{N}\right)=R_{N+1}-R_{N}-\lambda_{1}\left(\mathscr{E}_{N}\right) \leq \frac{1}{8}\left(\frac{3}{4}\right)^{N}, \quad N \in \mathbb{N}
$$

Consider two cases:

i) Let $r \in\left[1-\left(\frac{3}{4}\right)^{k}, 1-\frac{7}{6} \cdot\left(\frac{3}{4}\right)^{k+1}\right], k \in \mathbb{N}$. Then,

$$
\begin{aligned}
\lambda_{1}([r, 1) \cap F) & \leq \sum_{N=k}^{\infty} \lambda_{1}\left(\left[1-\left(\frac{3}{4}\right)^{N}, 1-\left(\frac{3}{4}\right)^{N+1}\right] \cap F_{N}\right)=\sum_{N=k}^{\infty} \lambda_{1}\left(F_{N}\right) \leq \\
& \leq \sum_{N=k}^{\infty} \frac{1}{8}\left(\frac{3}{4}\right)^{N}=\frac{1}{2}\left(\frac{3}{4}\right)^{k} .
\end{aligned}
$$

Since $r \leq 1-\frac{7}{6} \cdot\left(\frac{3}{4}\right)^{k+1}$, we obtain $\left(\frac{3}{4}\right)^{k} \leq \frac{8}{7}(1-r)$. Hence,

$$
\lambda_{1}([r, 1) \cap F) \leq \frac{4}{7}(1-r)
$$

ii) Let $r \in\left[1-\frac{7}{6} \cdot\left(\frac{3}{4}\right)^{k+1}, 1-\left(\frac{3}{4}\right)^{k+1}\right]$. Thus,

$$
\begin{aligned}
\lambda_{1}([r, 1) \cap F) & \leq \sum_{N=k+1}^{\infty} \lambda_{1}\left(F_{N}\right)+\frac{1}{6} \cdot\left(\frac{3}{4}\right)^{k+1} \\
& \leq \sum_{N=k+1}^{\infty} \frac{1}{8}\left(\frac{3}{4}\right)^{N}+\frac{1}{6} \cdot\left(\frac{3}{4}\right)^{k+1}=\frac{2}{3} \cdot\left(\frac{3}{4}\right)^{k+1} \leq \frac{2}{3}(1-r) .
\end{aligned}
$$

Therefore, we have proved that $\lambda_{1}([r, 1) \cap F) \leq \frac{2}{3}(1-r)$ holds for all $r<1$ sufficiently close to 1 . Thus, $D_{1}(F) \leq C \leq \frac{2}{3}$.

To complete the proof of Theorem 1 we need the following lemma. 
Lemma 3 [7] For arbitrary $\delta \in(0,1)$ and arbitrary $z \in \mathbb{D}$, the inequality

$$
|\log | P(z)\left|+N_{z}(\delta(1-|z|))\right| \leq C_{1}(\delta, s) \sum_{k=1}^{\infty}\left|A_{k}(z)\right|^{s+1}
$$

holds, where $C_{1}$ is a positive constant depending on $s$ and $\delta$.

From Lemmas 3 and 1, and (2) we get

$$
\begin{aligned}
\log |P(z)| & \geq-C_{1}\left(\frac{1}{4}, s\right) \sum_{k=1}^{\infty}\left|A_{k}(z)\right|^{s+1}-N_{z}\left(\frac{1}{4}(1-|z|)\right), \\
\log \mu(r, P) & \geq-\log \left(\frac{2 A}{1-r}\right)\left(\frac{1}{1-r}\right)^{\beta}-C_{2}\left(\frac{1}{4}, s, \beta\right)\left(\frac{1}{1-r}\right)^{\beta} \geq \\
& \geq-K\left(\frac{1}{1-r}\right)^{\beta} \log \frac{1}{1-r}, \quad r \nearrow 1, r \notin F
\end{aligned}
$$

where $K>1$ is an arbitrary constant. Theorem 1 is proved.

Proof of Proposition 1 Since $\left|u\left(r e^{i \theta}\right)\right| \leq M_{\infty}(r, u)$, we have $m_{p}(r, u) \leq M_{\infty}(r, u)$. It implies

$$
\rho_{\infty}[u] \leq \limsup _{r \nearrow 1} \frac{\log M_{\infty}(r, u)}{-\log (1-r)}:=\rho^{*}
$$

Let $P(z, w)=\operatorname{Re} \frac{w+z}{w-z}$ be the Poisson kernel. The Poisson formula together with Hölder's inequality yields

$$
\begin{aligned}
\left|u\left(r e^{i \theta}\right)\right| & \leq \frac{1}{2 \pi} \int_{0}^{2 \pi}\left|u\left(R e^{i \varphi}\right)\right| P\left(r e^{i \theta}, R e^{i \varphi}\right) d \varphi \\
& \leq\left(\frac{1}{2 \pi} \int_{0}^{2 \pi}\left|u\left(R e^{i \varphi}\right)\right|^{p} d \varphi\right)^{1 / p}\left(\frac{1}{2 \pi} \int_{0}^{2 \pi}\left(P\left(r e^{i \theta}, R e^{i \varphi}\right)\right)^{q} d \varphi\right)^{1 / q} \\
& =m_{p}(R, u)\left(\frac{1}{2 \pi} \int_{0}^{2 \pi}\left(P\left(r e^{i \theta}, R e^{i \varphi}\right)\right)^{\frac{p}{p-1}} d \varphi\right)^{\frac{p-1}{p}}
\end{aligned}
$$

where $\frac{1}{p}+\frac{1}{q}=1,0<r<R<1,0 \leq \theta<2 \pi$.

For the estimate of the Poisson kernel, we use the following lemma

Lemma 4 [10] If $a>0$ and $R=\frac{1}{2}(1+r)$ then

$$
\int_{0}^{2 \pi}\left|R e^{i t}-r\right|^{-a} d t=O\left((1-r)^{1-a}\right), r \nearrow 1 .
$$


Therefore,

$$
M_{\infty}(r, u) \leq K_{4} m_{p}(R, u)(1-r)^{-1 / p} \leq K_{4}\left(\frac{1}{1-R}\right)^{\rho_{p}+\varepsilon}(1-r)^{-1 / p}
$$

Putting $R=\frac{1}{2}(1+r)$, we get

$$
M_{\infty}(r, u) \leq K_{5}\left(\frac{1}{1-r}\right)^{\rho_{p}+\varepsilon+1 / p}
$$

Thus, as $p \rightarrow \infty$ we obtain

$$
\rho^{*} \leq \rho_{\infty}[u]+\varepsilon
$$

Hence, from arbitrariness of $\varepsilon>0$ it follows that $\rho^{*} \leq \rho_{\infty}[u]$, and finally

$$
\rho_{\infty}[u]=\limsup _{r \nearrow 1} \frac{\log M_{\infty}(r, u)}{-\log (1-r)} .
$$

Proof of Theorem 2 By Theorem F, we have

$$
f(z)=z^{p} P(z) g(z)
$$

where $P(z)$ is a canonical product of form (3) displaying the zeros of $f, p$ is a nonnegative integer, $g$ is non-zero, both $P$ and $g$ are analytic, and $\rho_{\infty}[P] \leq \rho, \rho_{\infty}[g] \leq \rho$. Further,

$$
\log |f(z)|=p \log |z|+\log |P(z)|+\log |g(z)| .
$$

Note that, by [6, Thm. 1.4]

$$
n_{z}\left(\frac{1-r}{2}\right) \leq(1-r)^{-\rho-\varepsilon},|z|=r \nearrow 1
$$

Applying Theorem 1 , with $\beta=\rho+\varepsilon$ we get

$$
\log \mu(r, P) \geq-K_{2}\left(\frac{1}{1-r}\right)^{\rho+\varepsilon}, r \in[0,1) \backslash F
$$

where $D_{1}(F) \leq C$.

We set $u=\log |g(z)|, u$ is harmonic in $\mathbb{D}$. Since $\rho_{M}[g] \leq \rho_{\infty}[g] \leq \rho$, applying Proposition 1, we deduce

$$
M_{\infty}(r, u) \leq(1-r)^{-(\rho+\varepsilon)}, \quad r \in\left[r_{0}, 1\right)
$$


which is equivalent to

$$
|\log | g\left(r e^{i \theta}\right)|| \leq(1-r)^{-(\rho+\varepsilon)} .
$$

Thus, we have

$$
\begin{aligned}
\log \mu(r, f) & \geq-(1-r)^{-(\rho+\varepsilon)}-K_{2}\left(\frac{1}{1-r}\right)^{\rho+\varepsilon} \log \left(\frac{1}{1-r}\right)+p \log r \\
& \geq-(1-r)^{-\rho-2 \varepsilon}, \quad r \nearrow 1, r \notin F
\end{aligned}
$$

Theorem 2 is proved.

\section{Generalizations}

Remark 4 One can replace the condition $n_{z}\left(\frac{1-|z|}{2}\right) \leq\left(\frac{1}{1-|z|}\right)^{\beta}$ in Theorem 1 by a more general one of the form $n_{z}\left(\frac{1-|z|}{2}\right) \leq \psi\left(\frac{1}{1-|z|}\right)$, where $\psi:[1,+\infty) \rightarrow \mathbb{R}_{+}$is a non-decreasing function such that $\psi(2 x)=O(\psi(x)), x \rightarrow \infty$. Then, one should replace the factor $\frac{1}{(1-r)^{\beta}} \log \frac{1}{1-r}$ by $\tilde{\psi}\left(\frac{1}{1-r}\right) \log \frac{1}{1-r}$ in the conclusion of Theorem 1 , where $\tilde{\psi}(x)=\int_{1}^{x} \frac{\psi(t)}{t} d t$ (see [7], for details).

If we have additional information on the factors in the factorization formula (4) we can state more, using the same method.

Theorem 3 Let $f$ be an analytic function in $\mathbb{D}$ of the form (4), $\psi$ is a positive nondecreasing function such that $\psi(2 x)=O(\psi(x))$ on $[1,+\infty)$. If

$$
M_{\infty}(r, \log |g|) \leq \psi\left(\frac{1}{1-r}\right)
$$

and the counting functions of the zeros of $f$ satisfy

$$
n_{z}\left(\frac{1-|z|}{2}\right) \leq \psi\left(\frac{1}{1-|z|}\right)
$$

then

$$
\log \mu(r, f) \geq-\tilde{\psi}\left(\frac{1}{1-r}\right) \log \frac{1}{1-r}
$$

where $\tilde{\psi}(x)=\int_{1}^{x} \frac{\psi(t)}{t} d t$

Acknowledgments We would like to thank the anonymous referee for careful reading of the manuscript and valuable remarks. 
Open Access This article is distributed under the terms of the Creative Commons Attribution License which permits any use, distribution, and reproduction in any medium, provided the original author(s) and the source are credited.

\section{References}

1. Barry, P.D.: On a theorem of Besicovitch. Q. J. Math. 14, 293-302 (1963)

2. Barry, P.D.: The minimum modulus of small integral and subharmonic functions. Proc. Lond. Math. Soc. 12, 445-495 (1962)

3. Ch. Berg, H.L. Pedersen, Logarithmic order and type of indeterminate moment problems. With an appendix by W. Hayman, Difference Equations, Special Functions and Orthogonal Polynomials: pp. 51-79 (2007)

4. Chyzhykov, I.: An addition to the $\cos \pi \rho$-theorem for subharmonic and entire functions of zero lower order. Proc. Am. Math. Soc. 130, 517-528 (2002)

5. Chyzhykov, I.: On minimum modulus of bounded analytic functions. Math. Bull. Shevchenko Scient. Soc. 4, 318-328 (2007)

6. Chyzhykov, I.: Zero distribution and factorisation of analytic functions of slow growth in the unit disc. Proc. Am. Math. Soc. 141, 1297-1311 (2013)

7. Chyzhykov, I., Sheparovych, I.: Interpolation of analytic functions of moderate growth in the unit disc and zeros of solutions of a linear differential equation. J. Math. Anal. Appl. 414, 319-333 (2014)

8. Chyzhykov, I., Skaskiv, S.: Growth, zero distribution and factorization of analytic functions of moderate growth in the unit disc, Blaschke products and their applications. Fields Inst. Comm. 65, 159-173 (2013)

9. Chyzhykov, I., Heittokangas, J., Rättyä, J.: Sharp logarithmic derivative estimates with applications to differential equations in the unit disc. J. Aust. Math. Soc. 88(2), 145-167 (2010)

10. Duren, P.: Theory of $H^{p}$ spaces. Academic Press, New York and London (1970)

11. Fenton, P.C.: The infimum of small subharmonic functions. Proc. Am. Math. Soc. 78(1), $43-47$ (1980)

12. Fenton, P.C.: The minimum of small entire functions. Proc. Am. Math. Soc. 81(4), 557-561 (1981)

13. Fenton, P.C.: The minimum modulus of certain small entire functions. Trans. Am. Math. Soc. 271(1), 183-195 (1982)

14. Fryntov, A.: A counterexample concerning the maximum and minimum of a subharmonic function. Proc. Am. Math. Soc. 122(4), 97-103 (1994)

15. Hayman, W.K.: The minimum modulus of large integral functions. Proc. London Math. Soc. 2, 469-512 (1952)

16. W.K. Hayman, Subharmonic functions, Vol. 2. London Math. Soc. Monogr. No.20, London, San Diego, New York, Berkeley, Boston, Sydney, Tokyo, Toronto 1989

17. Hayman, W.K.: The growth of bounded and realted functions. Bull. Lond. Math. Soc. 46, 537-549 (2014)

18. Heins, M.: The minimum modulus of a bounded analytic functions. Duke Math. J. 14, 179-215 (1947)

19. Juneja, O.P., Kapoor, G.P.: Analytic Functions - Growth Aspects, Research Notes in Mathematics 104. Pitman Adv. Publ. Prog, Boston-London-Melbourne (1985)

20. Linden, C.N.: The minimum modulus of functions regular and of finite order in the unit circle. Q. J. Math. 7, 196-216 (1956)

21. Linden, C.N.: The representation of regular functions. J. Lond. Math. Soc. 39, 19-30 (1964)

22. C.N.Linden, The minimum modulus of function of slow growth in the unit disk, Math. Essays dedicated to A. J. Macintyre, Ohio Univ. Press, 237-246 (1970)

23. Linden, C.N.: Integral logarithmic means for regular functions. Pac. J. Math. 138(1), 119-127 (1989)

24. Linden, C.N.: The characterization of orders for regular functions. Math. Proc. Cambrodge Philos. Soc. 111(2), 299-307 (1992) 